\title{
A standardized suppresses breast cancer cell proliferation by regulating the expression of EphA2 antisense RNA-mRNA axis independently of micro RNA
}

\author{
Ryou Sakamoto $^{1}$, Kazuhiro Kumagai ${ }^{1}$ Tokifumi Odaka $^{1}$, Tetsuya Okuyama ${ }^{2}$, Mikio \\ Nishizawa $^{2}$, and Tominori Kimura ${ }^{1}$
}

${ }^{1}$ Laboratory of Microbiology and Cell Biology, Department of Pharmaceutical Sciences, College of Pharmacy, Ritsumeikan University, Kusatsu, Shiga, 525-8577, Japan and ${ }^{2}$ Laboratory of Medical Chemistry, Department of Biomedical Sciences, College of Life Sciences, Ritsumeikan University, Kusatsu, Shiga, 525-8577, Japan

Corresponding author: Ryou Sakamoto, Laboratory of Microbiology and Cell Biology, Department of Pharmaceutical Sciences, College of Pharmacy, Ritsumeikan University, Kusatsu, Shiga, 525-8577, Japan

Submission Date: July $25^{\text {th }}$, 2019. Acceptance Date: September $27^{\text {th }}$, 2019. Publication Date: September $30^{\text {th }}, 2019$.

Citation: Sakamoto R., Kumagai K., Odaka T., Okuyama T., Nishizawa M., and Kimura T.. A standardized suppresses breast cancer cell proliferation by regulating the expression of EphA2 antisense RNA-mRNA axis independently of micro RNA. Bioactive Compounds in Health and Disease 2019; 2(9): 191-205. DOI: https://doi.org/10.31989/bchd.v2i9.642

\footnotetext{
ABSTRACT

Background: A standardized extract of cultured Lentinula edodes mycelia (ECLM), an extract from cultured Lentinula edodes, has been reported to suppress breast cancer stem cell proliferation by regulating microRNA (miR) expression. Natural antisense RNAs (ASs), a type of protein noncoding RNA, can regulate the expression of protein-coding genes by acting as a competing endogenous RNA (ceRNA) that adsorbs miRNAs, resulting in the prevention of mRNA degradation, and can also form a transient RNA duplex with mRNA. EphA2, a receptor tyrosine kinase, is typically expressed at low levels in normal epithelial cells, whereas its overexpression
} 
has been widely observed in numerous solid tumors and is associated with cell transformation, primary tumor initiation, and tumor progression.

Objective: This study aimed to investigate the effect of ECLM on the expression of both EphA2 mRNA and endogenous AS to this mRNA, which could negatively affect human breast carcinoma cell proliferation.

Methods: We used MCF7 and MDA-MB-231 human breast carcinoma cells, which were subcultured three times in the presence of optimized concentrations of ECLM. The effect of ECLM on the expression of EphA2 AS and mRNA was analyzed by RT-qPCR. miRNAs targeting both EphA2 AS and EphA2 mRNA and their RNA miR response elements (MREs) were predicted and analyzed by RT-qPCR and luciferase reporter assays.

Results: ECLM suppressed the proliferation of MCF7 and MDA-MB-231 cells in a dosedependent manner. In cells for which proliferation was negatively affected by ECLM, EphA2 AS and mRNA expression was also significantly inhibited by ECLM. Although neutralization of miR335 led to the de-repression of both EphA2 AS and mRNA, results did not fully support the possibility that EphA2 AS might function as a ceRNA to regulate EphA2 mRNA levels.

Conclusion: ECLM suppressed the proliferation of breast carcinoma cells in a specific dosedependent manner. This suppressive effect was associated with a concordant reduction in both EphA2 AS and mRNA expression. These effects were not thought to occur via the reported ceRNA effect. Therefore, these results suggest that ECLM suppresses the expression of EphA2 mRNA, which is regulated by EphA2 AS in a ceRNA-independent system, by suppressing the expression of EphA2 AS.

Keywords: ECLM, regulatory RNA, antisense RNA, microRNA, EphA2

\section{INTRODUCTION}

A standardized extract of cultured Lentinula edodes mycelia (ECLM) is an extract from cultured Lentinula edodes that reportedly exerts immune-protective effects against many types of cancer, including liver, breast, colon, and prostate [1-4]. In a recent study, ECLM was also reported to suppress breast cancer stem cell proliferation by regulating the expression of microRNAs (miRNAs) [5, 6]. These miRNAs are controlling genes involved in cellular processes such as inflammation, cell cycle regulation, stress response, differentiation, apoptosis, and migration [7]. 
Natural antisense RNAs (ASs), comprising a type of protein non-coding RNA, have been reported to regulate the expression of protein-coding genes by acting as a competing endogenous RNA (ceRNA) that adsorbs miRNAs resulting in the prevention of mRNA degradation; further, they can also form a transient RNA duplex [8-10].

EphA2, a receptor tyrosine kinase, is typically expressed at low levels in normal epithelial cells, whereas its overexpression has been widely observed in numerous solid tumors and is associated with cell transformation, primary tumor initiation, and tumor progression [11]. Therefore, we verified the possibility that ECLM regulated the expression of EphA2 mRNA by regulating EphA2 AS expression and suppresses breast cancer cell proliferation. We also verified the possibility that AS could function as a ceRNA for EphA2 as well.

\section{MATERIALS AND METHODS}

\section{Cell culture}

Human MDA-MB-231 cells (originating from mammary adenocarcinoma; ATCC HTB-26) were maintained in RPMI-1640 medium supplemented with $10 \%$ heat-inactivated fetal calf serum (FCS) (R10 medium). Human MCF7 cells (originating from mammary adenocarcinoma; ATCC HTB-22) were maintained in Dulbecco's Modified Eagle's Medium (D-MEM) containing 10\% FCS, $0.1 \mathrm{mM}$ non-essential amino acids, and $1 \mathrm{mM}$ sodium pyruvate (D10 medium).

To examine the effect(s) of ECLM (Amino Up Chemical, Sapporo, Japan) on EphA2 AS and mRNA expression levels and the potential effects as a ceRNA, MDA-MB-231 cells were maintained in R10 medium supplemented with this compound at $1.25 \mathrm{mg} / \mathrm{ml}, 0.625 \mathrm{mg} / \mathrm{ml}$ and 0 $\mathrm{mg} / \mathrm{ml}$; cells were sub-cultured three times. MCF7 cells were maintained in D10 medium supplemented with this compound at $0.625 \mathrm{mg} / \mathrm{ml}$ and $0 \mathrm{mg} / \mathrm{ml}$, and cells were also sub-cultured three times.

\section{Measurement of lactate dehydrogenase (LDH) activity}

As an indicator of cytotoxicity, LDH activity in the medium was measured in triplicate using LDH Cytotoxicity Detection Kits (Takara Bio, Otsu, Japan), according to the manufacturer's instructions.

\section{Oligonucleotides}

Unconjugated locked nucleic acid (LNA)-modified anti-miR-126, anti-miR-335, and anti-miR4267 oligonucleotides were synthesized with a complete phosphorothioate backbone (GeneDesign, Osaka, Japan). The sequence of antimiR-126, anti-miR-335, and anti-miR-4267 were 
complementary to the seed region of mature miR-126, miR-335, and miR-4267 (nucleotides 2-9), respectively. The LNA mismatch control was designed according to the miR-126 seed region sequence.

\section{Strand-specific reverse-transcription quantitative polymerase chain reaction (RT-qPCR)}

DNase I-treated total cellular RNA was annealed with strand-specific primers. cDNA was synthesized in both the presence and absence of $100 \mathrm{U}$ ReverTra Ace (TOYOBO, Osaka, Japan) at $50{ }^{\circ} \mathrm{C}$ for $30 \mathrm{~min}$. The cDNAs were then amplified by PCR using rTaq DNA Polymerase (TOYOBO) in a Thermal Cycler Dice Real Time System II (TaKaRa Bio, Shiga, Japan) using the following default thermocycler program: 1 min of pre-incubation at $95{ }^{\circ} \mathrm{C}$ followed by $22-38$ cycles of $15 \mathrm{~s}$ at $95{ }^{\circ} \mathrm{C}, 1 \mathrm{~min}$ at $72{ }^{\circ} \mathrm{C}$ (reducing the annealing temperature $0.3^{\circ} \mathrm{C}$ per cycle), and $30 \mathrm{~s}$ at $72{ }^{\circ} \mathrm{C}$. The primers used for RT and PCR of AS, mRNA, and 18S rRNA are shown in Table 1.

Table 1. List of primers used for RT-PCR

\section{For detection of EphA2 AS and mRNA}

RT primer for EphA2 AS: F, 5'-CGACATCAAGAGGATTGGGGTGCGG-3' (EphA2 mRNA NM_004431 nt 2981-3005)

RT primer for EphA2 AS: R, 5'-CGGTTTGAATCATCTGCAACTTTATTCC-3' (EphA2 mRNA nt 3963-3936)

PCR primer pair: F, 5'-CGACATCAAGAGGATTGGGGTGCGG-3' (EphA2 mRNA nt: 2981 -3005) / R, 5'- CAGCATCCCTGGTCATCTCCTCAGTTC-3' (EphA2 mRNA nt: 3271$3245)$

\section{For detection of $18 S$ rRNA}

RT primer for $18 \mathrm{~S}$ rRNA: a nona-deoxyribonucleotide random primer mixture (Takara Bio) PCR primer pair: F, 5'-CTTAGAGGGACAAGTGGCG-3' (human 18S rRNA nt1443-1461) / R, 5'-ACGCTGAGCCAGTCAGTGTA-3' (human 18S rRNA nt 1549-1529)

\section{RNA preparation and RNA-seq}

Total cellular RNA was isolated from MDA-MB-231 and MCF7 cells using Sepasol®-RNA I Super G (Nacalai tesque, Kyoto, Japan). RNA samples were then treated with proteinase K (Wako, Osaka, Japan) and TURBO DNA-free DNase I kit (Applied Biosystems, Carlsbad, USA) to remove RNase and genomic DNA.

For RNA-seq, ribosomal RNA was removed using the Ribo-Zero rRNA Removal Kit 
(Illumina, San Diego, USA), according to the manufacturer's instructions.

Sequence libraries were generated using TruSeq Stranded Total RNA Sample Prep Kit (Illumina). The libraries were then sequenced on an Illumina Hiseq 2500 platform, and 100-bp pair-end reads were generated. Sequence reads were analyzed for expression levels using the CLC genomics workbench (QIAGEN, Hilden, Germany).

\section{Plasmid construction}

To construct the pEF-luc-EphA2 AS reporter plasmid, EphA2 AS was amplified by PCR using pCG-EphA2 AS (accompanying paper) as a template. EphA2 AS was digested with XbaI and ligated into the $X b a \mathrm{I}$ site of the pEF-luc-SVpA [12] reporter vector.

To construct the pEF-luc-EphA2 AS miR response element (MRE)-126 seed revertant (SR), MRE-335SR, and MRE-4267SR, PCR and the Gibson assembly system were employed using the Q5 High-Fidelity DNA Polymerase (New England Biolabs, Ipswich, USA) and Gibson Assembly Master Mix (New England Biolabs, Ipswich, USA), according to the manufacturer's instructions. Primers for construction of the above vectors are shown in Table 2. These MRE revertants were changed MRE sequences to these complementary sequences.

The stem loop sequences of Homo sapiens miR-126, miR-335, and miR-4267 (DDBJ/EMBL/GenBank Accession Numbers: MI0000471, MI0000816, and MI0015871, respectively) were retrieved from the miRBase database. DNA fragments containing 100 bases of both upstream and downstream native flank sequence of each miRNA stem loop were then amplified by PCR and cloned into the BamHI and NheI sites of the pEGFP-miR expression vector (Cell Biolabs, San Diego, CA, USA) to create pEGFP-miR-126, pEGFP-miR-335, and pEGFPmiR-4267. pLKO-miR-126, pLKO-miR-335, and pLKO-miR-4267 lentiviral vectors were then constructed by replacing the blunted XhoI and KpnI fragments of shTORC2 containing the U6 promoter-shTORC2 complementary DNA (cDNA) with a blunted ClaI and KpnI fragment of pEGFP-miR-126, pEGFP-miR-335, and pEGFP-miR-4267 containing EF-1 $\alpha$ promoter-miRNA126, EF-1 $\alpha$ promoter-miR-335, and EF-1 $\alpha$ promoter-miR-4267 or the same fragment from the pEGFP-miR-null control vector (Cell Biolabs). Recombinant lentiviruses encoding miR-126, miR-335, miR-4267, and miR-null were generated in HEK293T cells (ATCC CRL-1126) through the co-transfection of pLKO-miR-126, pLKO-miR-335, and pLKO-miR-4267 or pLKO-null with the packaging constructs $\mathrm{pCMV} \Delta \mathrm{R} 8.2$ and $\mathrm{pVSV}-\mathrm{G}$.

\section{Transfection and lentiviral transduction}

MDA-MB-23 1 cells were transfected using Lipofectamine ${ }^{\circledR} 3000$ reagent. $2.2 \mu \mathrm{g}$ of plasmids were mixed with P3000 regent and Lipofectamine ${ }^{\circledR} 3000$ reagent was added. After a 15 -min incubation, 
the mixed regent was added to MDA-MB-231 cells, which were transferred to a $\mathrm{CO}_{2}$ incubator for $24 \mathrm{~h}$ at $37^{\circ} \mathrm{C}$.

For transduction with lentiviral vectors, viral supernatants were collected $48 \mathrm{~h}$ after transfecting HEK293T cells with pLKO-miR-126, pLKO-miR-335, and pLKO-miR-4267 or pLKO-null and MDA-MB-231 cells were transduced with the recombinant lentiviruses. The transduced cells were then selected with puromycin $(2 \mu \mathrm{g} / \mathrm{ml}$; Sigma-Aldrich, St. Louis, MO, USA). For the transfection of anti-miR, $1 \mu \mathrm{g}$ of anti-miR was transfected as described.

Table 2. Nucleotide sequences of primers for the construction of miR response element mutant reporters

For construction of pEF-luc-EphA2 AS

F, 5'-gctctagaGAGGATGGGGCCCGAGGG-3' (EphA2 mRNA nt 3589-3572)

R, 5'-gctctagaCCCACACCCATGGACTGCCC-3' (EphA2 mRNA nt 2664-2683)

For construction of pEF-luc-EphA2 AS MRE-126 SR

Fragment1

F, 5'-TCAGAGAGATCCTCATAAAGGCCAA-3' (U47295.2 Cloning vector pGL3-Basic nt1691-1715)

R, 5'-GCAGcatggcaCACGTCCCAGCATCCCTG-3' (EphA2 mRNA nt3317-3345)

Fragment2

F, 5'-GCTGGGACGTGtgccatgCTGCTAAGTGCTCAGCT-3' (EphA2 mRNA nt3338-3304)

R, 5'-CATTCTAGTTGTGGTTTGTCCAAACTC-3' (U47295.2 Cloning vector pGL3-Basic nt1821-1795)

For construction of pEF-luc-EphA2 AS MRE-335 SR

Fragment1

F, 5'-TCAGAGAGATCCTCATAAAGGCCAA-3' (U47295.2 Cloning vector pGL3-Basic nt1691-1715)

R, 5'-CGACAagttctcGATTGGGGTGCGGCTGCC-3' (EphA2 mRNA nt2981-3009)

Fragment2

F, 5'-GCACCCCAATCgagaactTGTCGTCGTTGGTCATC-3' (EphA2 mRNA nt3003-2969)

R, 5'-CATTCTAGTTGTGGTTTGTCCAAACTC-3' (U47295.2 Cloning vector pGL3-Basic nt1821-1795)

For construction of pEF-luc-EphA2 AS MRE-4267 SR

Fragment1

F, 5'-TCAGAGAGATCCTCATAAAGGCCAA-3' (U47295.2 Cloning vector pGL3-Basic nt1691-1715)

R, 5'-CATCTAggtcgagATGATGCAGTGCTGGCAGCAG-3' (EphA2 mRNA nt 2690-2723)

Fragment2

F, 5'-CACTGCATCATctcgaccTAGATGGCGGAGGGGCA-3' (EphA2 mRNA nt 2713-2679)

R, 5'-CATTCTAGTTGTGGTTTGTCCAAACTC-3' (U47295.2 Cloning vector pGL3-Basic nt1821-1795) 


\section{Luciferase reporter assays}

To analyze the effect of miRNA and ECLM on EphA2 AS and mRNA expression, we performed luciferase reporter assays. For reporter gene assays, $6.2 \times 10^{5}$ MDA-MB-231 cells/well were transfected with a luciferase reporter plasmid $(0.3 \mu \mathrm{g} /$ well) using Lipofectamine 3000 (Invitrogen, Carlsbad, USA). Cells were co-transfected with the pRL-RSV plasmid encoding Renilla luciferase $(0.8 \mu \mathrm{g} /$ well $)$, to control for differences in transfection efficiency.

Firefly and Renilla luciferase activities were measured using the Dual-Luciferase Reporter Assay System (Promega), according to the manufacturer's instruction. Protein concentrations were measured using the Protein Assay BCA Kit (Wako, Osaka, Japan). Firefly luciferase activity was normalized to Renilla luciferase activity and the amount of total protein.

\section{Statistical analysis and informatics}

Differences presented in the figures were analyzed by performing a Student's $t$ test.

\section{RESULTS}

\section{ECLM suppresses the proliferation of human breast cancer cells}

We first investigated whether ECLM inhibits proliferation in breast cancer cells by cell counting. ECLM significantly suppressed proliferation at concentrations of $0.625 \mathrm{mg} / \mathrm{ml}$ for MCF7 cells (48 hr) and $1.25 \mathrm{mg} / \mathrm{ml}$ for MDA-MB-231 cells (48-72 hr). These results indicated that ECLM functions during periods of the low confluence with poor cell adhesion. In addition, ECLM at a concentration of $1.25 \mathrm{mg} / \mathrm{ml}$ completely inhibited the growth of MCF7 cells (data not shown). The cytotoxicity of ECLM toward MDA-MB-231 cells was observed $24 \mathrm{~h}$ after sub-culture, but not after $48 \mathrm{~h}$ of sub-culture, at which time cell proliferation was suppressed (Fig.1).

\section{Identification of a naturally occurring antisense transcript overlapping human $\mathrm{EPHA2}$}

To determine whether EphA2 AS is expressed in MDA-MB-231 cells we employed RNA-seq. This revealed the presence of reads corresponding to the opposite strand of EPHA2, which were widely distributed in the region overlapping mRNA exon 1-17, and included the overlapping EphA2 mRNA3'UTR. (Table 3).

\section{Effect of ECLM on EphA2 antisense and mRNA levels}

We next examined the effect of ECLM on the expression levels of EphA2 AS and mRNA in MDAMB-231 and MCF7 cells. Levels of EphA2 AS and mRNA were concordantly suppressed by ECLM in MDA-MB-231 (accompanying paper) and MCF7 cells (Fig. 2). 


\section{EphA2 antisense regulates the miRNA-independent expression of EphA2 mRNA}

A recent study showed that AS sequences sharing miRNA response elements (MREs) with coding transcripts can be similarly targeted, resulting in the sequestering of miRNAs to prevent them from acting on mRNAs. Bioinformatics analysis (RegRNA [13], RNAhybrid [14], miRDB [15], and PITA [16]) was performed to predict the MREs that are shared by EphA2 AS and EphA2 mRNA 3'UTR, and these were predicted to share several MREs (MRE-126, MRE-335, and MRE-4267; Fig.3).
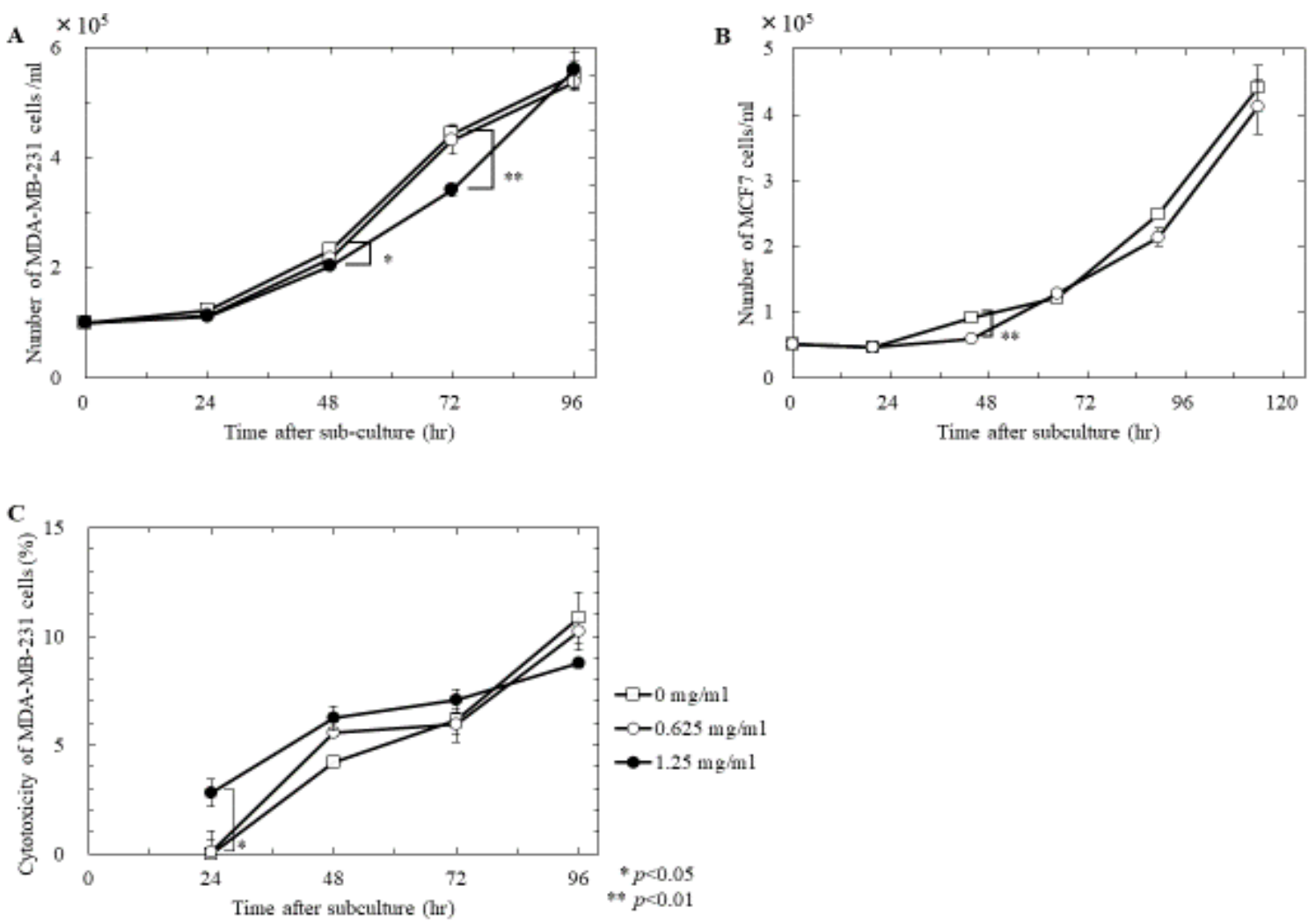

Figure 1. The effects of ECLM on breast cancer cell proliferation and cytotoxicity. A, B. Growth curves of MDA-MB-231 (A) and MCF7 (B) cells, as assessed by cell counting. C. Cytotoxicity of ECLM toward MDA-MB-231 cells, as assessed by LDH assays.

\section{Validation of miR-126, miR-335, and miR-4267 MREs in EphA2 AS and mRNA}

To validate the presence of miR-126, miR-335, and miR-4267 MREs in EphA2 AS and mRNA, we assessed the effect of endogenous miR-126, miR-335, and miR-4267 expression on EphA2 AS and mRNA levels by employing an anti-miR approach to inhibit endogenous miR expression[17]. For this knockdown strategy, we designed 8-mer anti-miR-126, miR-335, and miR-4267 sequences, using a fully LNA-modified phosphorothioate oligonucleotide complementary to the seed region of these miRNAs. We then tested their effects on EphA2 AS and mRNA expression in 
non-ECLM treated MDA-MB-231 cells. Silencing endogenous miR-335 increased EphA2 AS and mRNA levels by 36- and 28-fold, respectively, compared to expression with an LNA mismatch control (Fig. 4).

Table 3. Positions of EphA2 antisense reads in EPHA2

\begin{tabular}{|c|c|c|c|c|c|c|}
\hline \multicolumn{3}{|c|}{ Position of EphA2 AS reads in EPHA2 } & \multicolumn{4}{|c|}{ Position of EphA2 AS reads in EphA2 mRNA } \\
\hline 31,650 & - & 31,538 & 3,863 & - & 3,751 & exon17 \\
\hline 31,188 & - & 31,088 & 3,401 & - & 3,301 & exon 17 \\
\hline 30,416 & - & 30,362 & & & & intron 16 \\
\hline 26,654 & - & 26,568 & 2,980 & - & 2,894 & exon16 \\
\hline 24,249 & - & 23,898 & & & & exon $14-13$ \\
\hline 23,891 & - & 23,679 & & & & exon $13-12$ \\
\hline 22,796 & - & 22,203 & 2,096 & - & 1,868 & exon11-9 \\
\hline 20,975 & - & 20,389 & 1,660 & - & 1,539 & exon7-6 \\
\hline 20,621 & - & 20,537 & & & & intron6 \\
\hline 18,213 & - & 17,983 & 1,445 & - & 1,215 & exon5 \\
\hline 16,004 & - & 15,749 & & & & intron3 \\
\hline 15,567 & - & 15,480 & & & & intron3 \\
\hline 14,110 & - & 13,998 & & & & intron3 \\
\hline 11,740 & - & 11,494 & & & & intron3 \\
\hline 11,134 & - & 11,024 & & & & intron3 \\
\hline 10,291 & - & 10,184 & & & & intron3 \\
\hline 10,125 & - & 10,027 & & & & intron3 \\
\hline 9,850 & - & 9,703 & & & & intron3 \\
\hline 9,382 & - & 9,251 & & & & intron3 \\
\hline 9,123 & - & 8,961 & & & & intron3 \\
\hline 8,864 & - & 8,681 & & & & intron3 \\
\hline 8,575 & - & 8,383 & & & & intron3 \\
\hline 7,358 & - & 7,194 & 626 & - & 462 & exon3 \\
\hline 4,268 & - & 4,123 & & & & intron1 \\
\hline 4,103 & - & 3,995 & & & & intron1 \\
\hline 3,761 & - & 3,637 & & & & intron1 \\
\hline 3,350 & - & 3,154 & & & & intron1 \\
\hline 2,920 & - & 2,782 & & & & intron1 \\
\hline 1,829 & - & 1,648 & & & & intron1 \\
\hline 944 & - & 290 & & & & intron1 \\
\hline 198 & - & 25 & 198 & - & 25 & exon1 \\
\hline
\end{tabular}


A

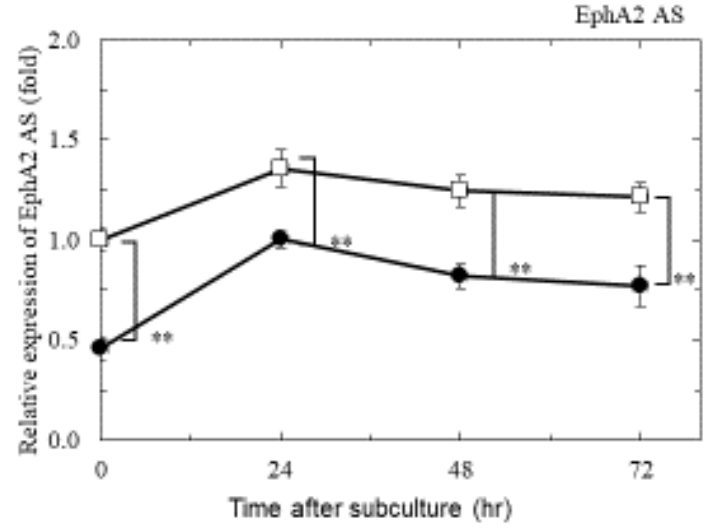

B

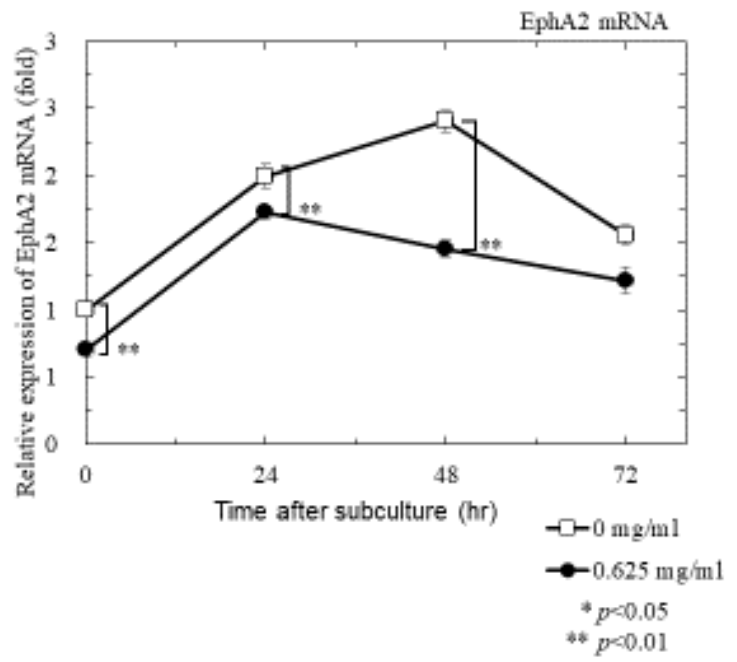

Figure 2. Effect of ECLM on EphA2 antisense (AS) and mRNA levels in MCF7 cells. Shown are expression levels of EphA2 AS (A) and EphA2 mRNA (B).

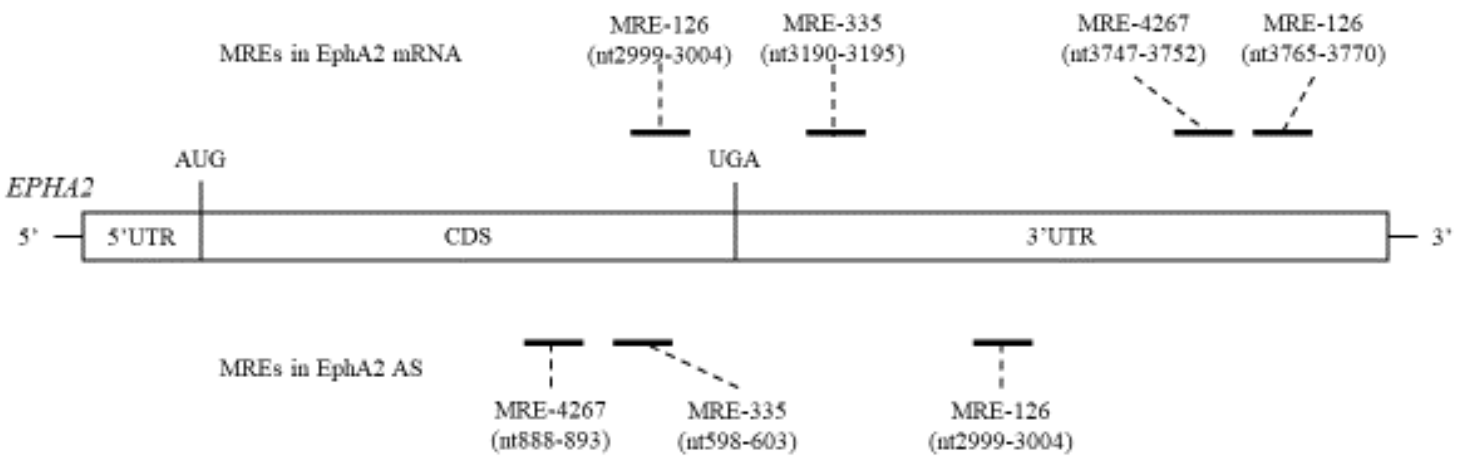

Figure 3. Predicted miRNA response elements (MREs) shared by EphA2 antisense (AS) and EphA2 mRNA 3'UTR. Several MREs (MRE-126, -MRE335, and MRE-4267) in EphA2 AS (reverse strand of mRNA) and EphA2 mRNA 3'UTR were predicted.

We next examined the effect of miR-126, miR-335, and miR-4267 on EphA2 AS and mRNA levels by transducing MDA-MB-231 cells with the virus to overexpress these miRNAs. Contrary to the results of the miR-335-neutralization experiment, suppression of EphA2 AS and mRNA after transfection of the predicted miRNA overexpression construct was not observed by RT-qPCR and luciferase reporter assays (Fig. 5).

To verify specific MRE-miRNA interactions, we used MRE-inverted luciferase reporters. Inverse MRE-126, MRE-335, and MRE-4267 sequences in EphA2 AS were inserted downstream of the luciferase reporter gene and their effects were tested on luciferase reporter activity using pLKO-miR-126, pLKO-miR-335, and pLKO-miR-4267 virus-transduced cells (Fig. 6). These 
transduced cells were overexpressed these miRs (data not shown). MiR-126, -335, and -4267 MRE-inverted luciferase reporters in the corresponding miRNA transduced cell, there are no increase in luciferase activity was observed, indicating that these miRNAs did not affect EphA2 AS expression. In addition, similar results was obtained for miR-126, -335, and -4267 MRE triple-inverted luciferase reporter in the parental cell.

A

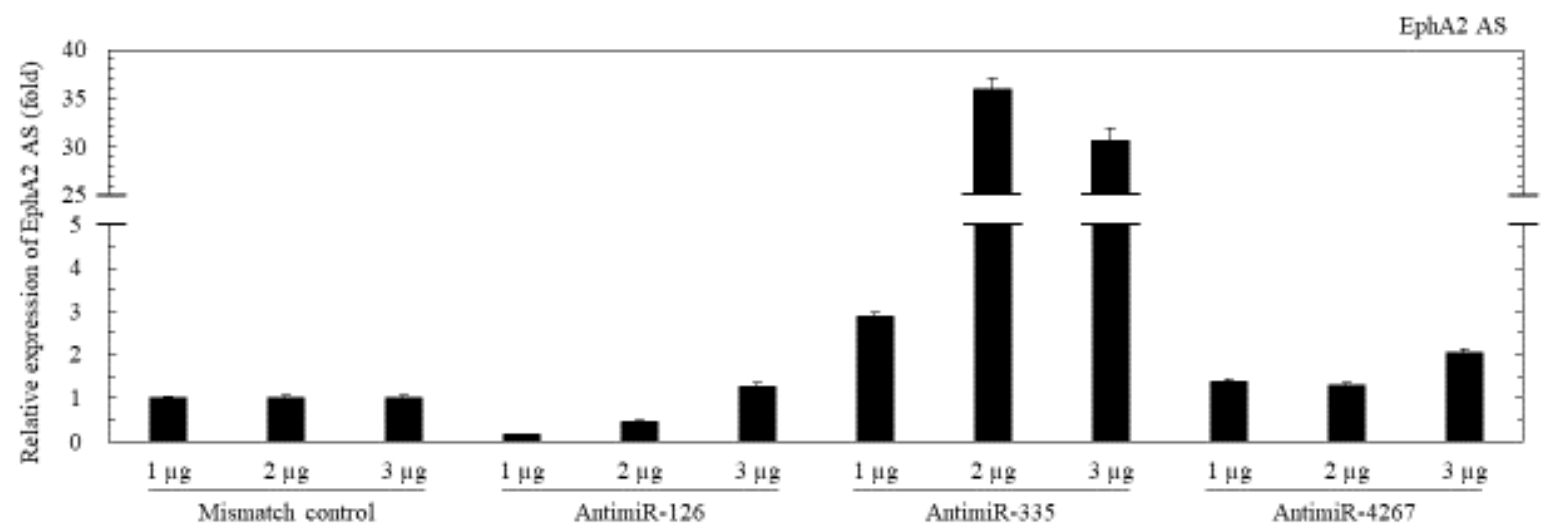

B

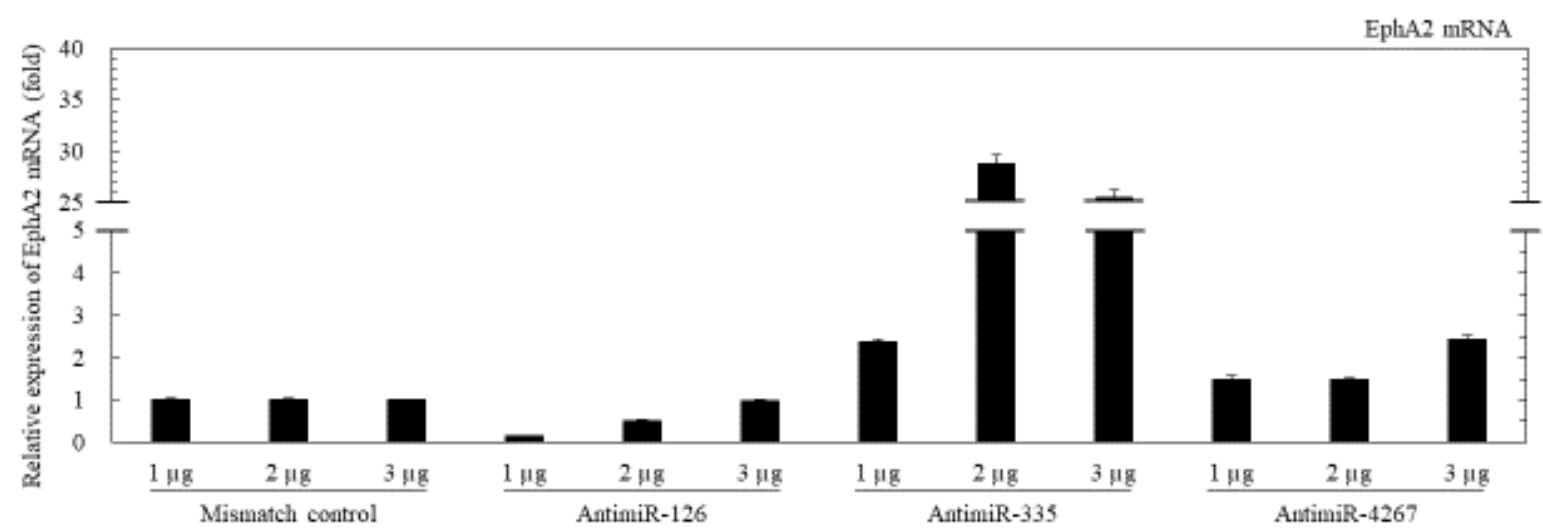

Figure 4. Effect of miR-126, miR-335, and miR-4267 neutralization on EphA2 antisense (AS)/mRNA levels. LNA-modified anti-miR-126, anti-miR-335, and anti-miR-4267 were transfected into MDA-MB-231 cells, which were harvested for the RT-qPCR analysis of relative EphA2 AS (A) and EphA2 mRNA (B) expression. The relative expression was presented as the fold-change in AS and mRNA levels relative to those obtained in LNA mismatch controltransfected cells.

\section{DISCUSSION}

ECLM was previously reported to have various anti-cancer effects via an immune-protective effect and through the regulation of miRNA expression [1-5]. ECLM is also known to reduce the risk of adverse neutrophil events in breast cancer patients who received adjuvant chemotherapy at $3 \mathrm{~g} /$ day 
oral administration [2]. These reports suggested that ECLM is useful for both the treatment of breast cancer and the prevention of chemotherapy side effects.
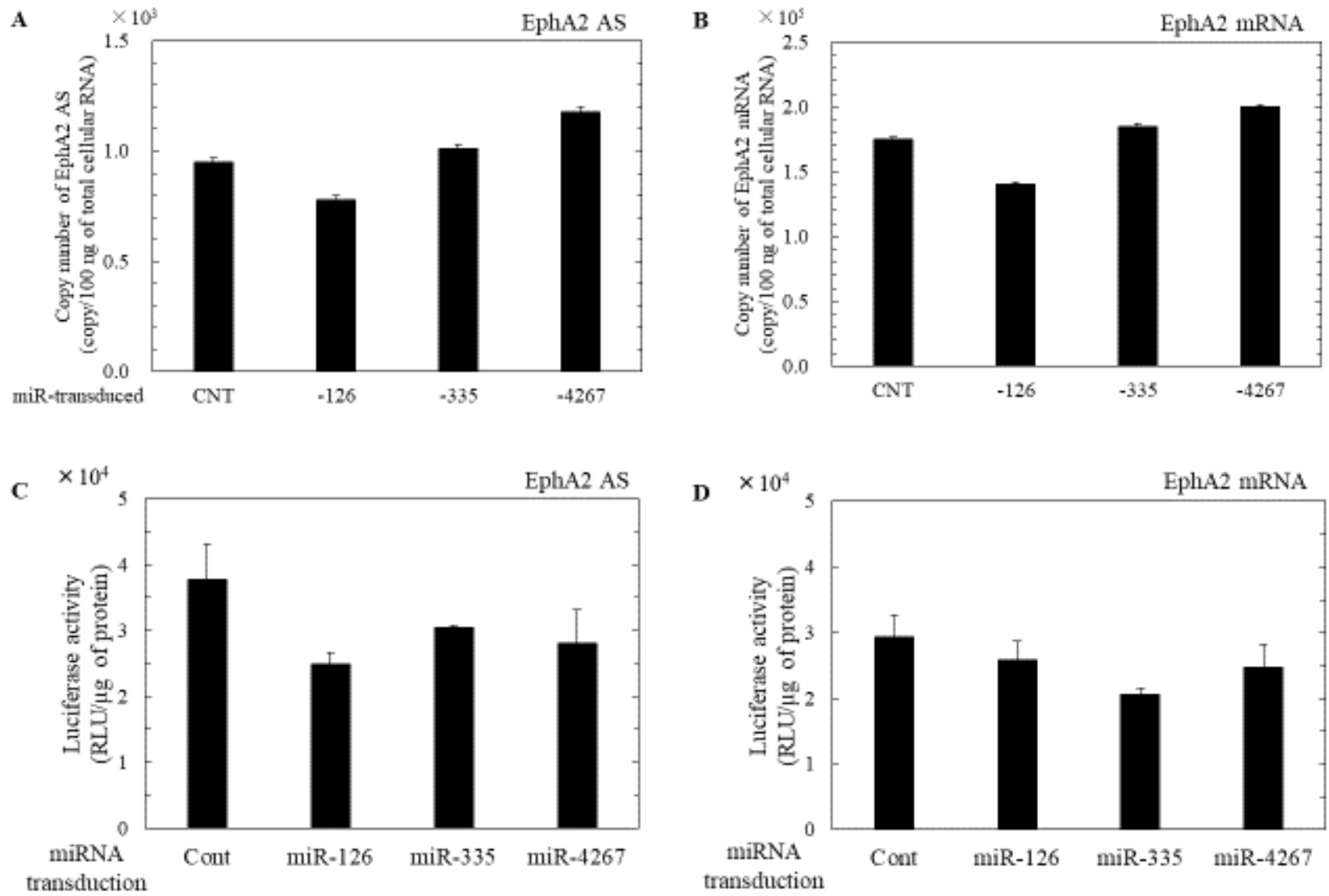

Figure 5. Validation of the effect of miR-126, miR-335, and miR-4267 on EphA2 antisense (AS)/mRNA levels. Effects miR-126, miR-335, and miR-4267 overexpression on EphA2 AS and mRNA expression were examined after transduction into MDA-MB-231 cells. The cells were then harvested for the RT-qPCR analysis of relative EphA2 AS (A) and EphA2 mRNA (B); luciferase reporter assays were also performed for EphA2 AS (C) and EphA2 mRNA(D) activity.

EphA2, which is highly expressed in triple-negative breast cancer, forms a heterodimer with EGFR and is involved in cell proliferation [18]. Accordingly, the delivery of EphrinA1 to tumor tissues, which is involved in the suppression of EphA2, as well as methods using siRNA against EphA2 and antibodies have been examined [19-21]. Compared to these methods, the suppression of breast cancer cell proliferation by inhibiting of EphA2 AS and mRNA expression via ECLM administration is advantageous in that the oral administration of this compound is and safe.

The possibility that EphA2 AS functions as a ceRNA to protect mRNA could be confirmed by miR-335 neutralization experiments; however, the negative results obtained herein did not support this effect. Therefore, it is thought that there is a regulating mechanism by non-ceRNA effect 
including mRNA stabilization through sense-antisense duplex formation reported [8]..
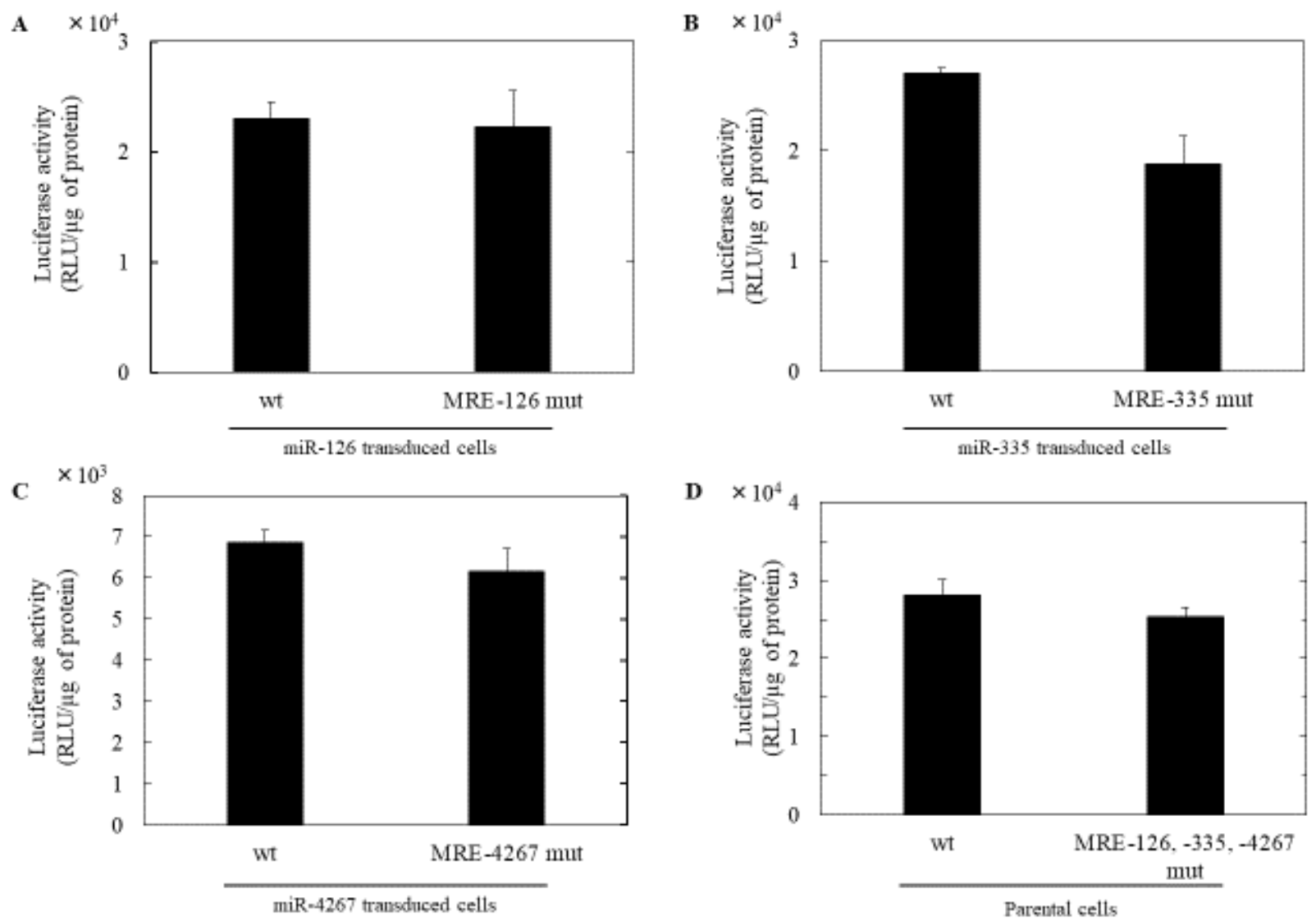

Figure 6. Validation of the effect of inverse miRNA response element (MRE)-126, MRE-335, and MRE-4267 on EphA2 antisense (AS). The effects of miRNA-MRE on EphA2 AS levels were analyzed using an MRE-126 seed revertant (SR) in miR-126-transduced cells (A), an MRE-335 SR in miR-335-transduced cells (B), an MRE-4267 SR in miR-4267-transduced cells (C), and MRE-126, MRE-335, and MRE-4267 SRs in parental cells (D).

\section{CONCLUSION}

ECLM suppresses the proliferation of breast carcinoma cells in a specific dose-dependent manner and inhibits both EphA2 AS and mRNA expression. However, this regulatory event did not appear to occur through a ceRNA effect. These results thus suggest that ECLM could regulate EphA2 AS and mRNA expression by not ceRNA effect, thereby stabilizing EphA2 mRNA. Therefore, it is necessary to further examine this regulation mechanism.

\section{Acknowledgements}

We are grateful to Noriyuki Yoshida who participated in early stages of this project and to all the present members of the Kimura laboratory for stimulating discussions. 


\section{REFERENCES}

1. Cowawintaweewat S, Manoromana S, Sriplung H, Khuhaprema T, Tongtawe P, Tapchaisri P, Chaicumpa W: Prognostic improvement of patients with advanced liver cancer after active hexose correlated compound (AHCC) treatment. Asian Pac J Allergy Immunol 2006, 24:33-45.

2. Hangai S, Iwase S, Kawaguchi T, Kogure Y, Miyaji T, Matsunaga T, Nagumo Y, et al.: Effect of active hexose-correlated compound in women receiving adjuvant chemotherapy for breast cancer: a retrospective study. J Altern Complement Med 2013, 19:905-10.

3. Turner J, Chaudhary U: Dramatic prostate-specific antigen response with activated hemicellulose compound in metastatic castration-resistant prostate cancer. Anticancer Drugs 2009, 20:215-6.

4. Hirose A, Sato E, Fujii H, Sun B, Nishioka H, Aruoma OI: The influence of active hexose correlated compound (AHCC) on cisplatin-evoked chemotherapeutic and side effects in tumor-bearing mice. Toxicol Appl Pharmacol 2007, 222:152-8.

5. Graham EA, Mallet JF, Jambi M, Nishioka H, Homma K, Matar C: MicroRNA signature in the chemoprevention of functionally-enriched stem and progenitor pools (FESPP) by Active Hexose Correlated Compound (AHCC). Cancer Biol Ther 2017, 18:765-774.

6. Bartel DP: MicroRNAs: target recognition and regulatory functions. Cell 2009, 136:21533.

7. Di Leva G, Garofalo M, Croce, CM: MicroRNAs in cancer. Annu Rev Pathol 2014, 9:287-314.

8. Nishizawa M, Ikeya Y, Okumura T, Kimura T: Post-transcriptional inducible gene regulation by natural antisense RNA. Front Biosci (Landmark Ed) 2015, 20:1-36.

9. Kimura T, Jiang S, Nishizawa M, Yoshigai E, Hashimoto I, Nishikawa M, Okumura T, et al.: Stabilization of human interferon-alpha1 mRNA by its antisense RNA. Cell Mol Life Sci 2013, 70:1451-67.

10. Kimura T, Jiang S, Yoshida N, Sakamoto R, Nishizawa, M: Interferon-alpha competing endogenous RNA network antagonizes microRNA-1270. Cell Mol Life Sci 2015, 72:2749-61.

11. Park JE, Son AI, Zhou, R: Roles of EphA2 in Development and Disease. Genes (Basel) 2013, 4:334-57. 
12. Matsui K, Nishizawa M, Ozaki T, Kimura T, Hashimoto I, Yamada M, Kaibori M, et al.: Natural antisense transcript stabilizes inducible nitric oxide synthase messenger RNA in rat hepatocytes. Hepatology 2008, 47:686-97.

13. Chang TH, Huang HY, Hsu JB, Weng SL, Horng JT, Huang HD: An enhanced computational platform for investigating the roles of regulatory RNA and for identifying functional RNA motifs. BMC Bioinformatics 2013, 14 Suppl 2:S4.

14. Rehmsmeier M, Steffen P, Hochsmann M, Giegerich R: Fast and effective prediction of microRNA/target duplexes. RNA 2004, 10:1507-17.

15. Wong N, Wang X: miRDB: an online resource for microRNA target prediction and functional annotations. Nucleic Acids Res 2015, 43:D146-52.

16. Kertesz M, Iovino N, Unnerstall U, Gaul U, Segal E: The role of site accessibility in microRNA target recognition. Nat Genet 2007, 39:1278-84.

17. Obad S, Dos Santos CO, Petri A, Heidenblad M, Broom O, Ruse C, Fu C, et al.: Silencing of microRNA families by seed-targeting tiny LNAs. Nat Genet 2011, 43:371-8.

18. Song W, Hwang Y, Youngblood VM, Cook RS, Balko JM, Chen J, Brantley-Sieders DM: Targeting EphA2 impairs cell cycle progression and growth of basal-like/triple-negative breast cancers. Oncogene 2017, 36:5620-5630.

19. Noblitt LW, Bangari DS, Shukla S, Knapp DW, Mohammed S, Kinch MS, Mittal SK: Decreased tumorigenic potential of EphA2-overexpressing breast cancer cells following treatment with adenoviral vectors that express EphrinA1. Cancer Gene Ther 2004, 11:757-66.

20. Carles-Kinch K, Kilpatrick KE, Stewart JC, Kinch MS: Antibody targeting of the EphA2 tyrosine kinase inhibits malignant cell behavior. Cancer Res 2002, 62:2840-7.

21. Ozcan G, Ozpolat B, Coleman RL, Sood AK, Lopez-Berestein G: Preclinical and clinical development of siRNA-based therapeutics. Adv Drug Deliv Rev 2015, 87:108-19. 